\title{
LA RELACIÓN ENTRE FOCO Y PROSODIA: ANÁLISIS DE LA PERCEPCIÓN DE LAS PROMINENCIAS ACENTUALES EN UN CORPUS DEL ESPAÑOL DE BUENOS AIRES
}

\section{THE RELATIONSHIP BETWEEN FOCUS AND PROSODY: ANALYSIS OF ACCENTUAL PROMINENCE PERCEPTION IN A SPANISH FROM BUENOS AIRES CORPUS}

\author{
Mercedes GÜEMES ${ }^{2,3}$ \\ BÁRBARA SAMPEDRO ${ }^{2,3,4}$ \\ Christian Cossio-Mercado ${ }^{1,2,5}$ \\ JORGE GURLEKIAN ${ }^{1,2}$ \\ anagraf99@hotmail.com
}

Recibido: 27/01/2016

Aceptado: 21/04/2016

\section{Resumen}

Todas las lenguas presentan formas de marcar el foco para diferenciarlo de los patrones no-marcados. La prosodia constituye una herramienta esencial para la producción y percepción del foco oracional. El objetivo del presente trabajo es analizar la identificación del foco y sus asociaciones con la percepción de la prominencia. Se realizó un experimento con 540 oraciones afirmativas e interrogativas, y con distintas variaciones focales (sujeto, predicado y foco neutro). Siete hablantes del español definieron el tipo de oración, identificaron el tipo de foco y marcaron el nivel de prominencia para cada una de las sílabas de la oración. Los resultados
\end{abstract}

\begin{abstract}
All languages have ways of designate the focus to differentiate it from unmarked patterns. Prosody is an essential tool for the production and perception of focus. The aim of this paper is to analyze the focus identification and its associations with perceived prominence. It was made an experiment with 540 affirmative and interrogative sentences, and with different focal variations (subject, predicate and neutral focus). Seven Spanish speakers defined the sentence modality, the type of focus and the level of prominence for each syllable of the sentence. Results show that all the speakers defined the type of sentence correctly. However, a high
\end{abstract}

\footnotetext{
1 Laboratorio de Investigaciones Sensoriales LIS-INIGEM

2 Consejo Nacional de Investigaciones Científicas y Técnicas, Argentina

3 Facultad de Filosofía y Letras - Universidad de Buenos Aires, Argentina

4 Facultad de Psicología - Universidad de Buenos Aires, Argentina

5 Facultad de Ciencias Exactas y Naturales - Universidad de Buenos Aires, Argentina
}

Para citar este artículo / To cite this article: Güemes, Mercedes; Sampedro, Bárbara; CossioMercado, Christian; Gurlekian, Jorge (2016). La relación entre foco y prosodia: análisis de la percepción de las prominencias acentuales en un corpus del español de Buenos Aires. ELUA, 30: 129-139. doi:10.14198/ELUA2016.30.06

Enlace / Link: http://dx.doi.org/10.14198/ELUA2016.30.06 
muestran que la totalidad de los hablantes definen de manera correcta el tipo de oración. Sin embargo, un alto porcentaje de evaluadores percibió con foco en el predicado a las oraciones emitidas con foco neutro. Además, se observó que las curvas de prominencia tonal no siempre correlacionaron con la producción de foco.

PALABRAS CLAVE: foco, prosodia, percepción, prominencia acentual. percentage of subjects perceived neutral focus utterances as with focus on the predicate. Also it was noted that tonal curves prominence not always correlate with focus production.

KEYWORDS: focus, prosody, perception, accentual prominence.

\section{Introducción}

Las estructuras lingüísticas comprenden las propiedades necesarias para hacerlas unidades comunicativas. Si bien cada lengua tiene sus formas de marcar el foco para diferenciarlo de los patrones no-marcados, todas las lenguas tienen la capacidad de codificarlo, lo que lo constituye un fenómeno pragmático universal. La marcación del foco puede deberse a distintas motivaciones (transmisión de información nueva, razones estilísticas, intereses comunicativos) y se manifiesta mediante diversos recursos (acentuales, léxicos, sintácticos). De esta manera, Dik (1997) define el foco como información saliente en su codificación y relevante en relación con las necesidades informativas del destinatario. Si bien las clasificaciones del foco son diversas y se desprenden de distintas perspectivas teóricas, cabe señalar que la mayoría de ellas considera el contexto como el generador del foco.

Zubizarreta (1999) especifica dos clases de foco sobre la base de su contexto: neutro o contrastivo. El foco neutro se identifica por medio de una pregunta con diversos alcances (contexto interrogativo). El ejemplo (1.a), de acuerdo con su alcance informativo, puede responder a (1.b), (1.c) o (1.d) según si se indaga por la totalidad del hecho, la predicación sobre un sujeto o un aspecto específico del predicado, respectivamente.

(1) a. El gato se comió un ratón.

b. ¿Qué ocurrió?

c. ¿Qué hizo el gato?

d. ¿Qué se comió el gato?

En cambio, el foco contrastivo niega una presuposición (contexto asertivo) como en el ejemplo (2).

(2) El gato se comió un ratón (no un canario).

A su vez, cada uno de estos focos está relacionado con dos tipos de acento, el foco neutro con acento nuclear neutro que se coloca en la última palabra del grupo o constituyente melódico, y el contrastivo con acento enfático que subraya algunos sintagmas.

Por otra parte, Dorta (2008) resume las definiciones de foco de acuerdo a la extensión del segmento focalizado. De esta manera, el foco neutro (Halliday, 1967) corresponde a toda la oración, el foco amplio a un constituyente oracional y el foco estrecho a un elemento léxico 
(Ladd, 1983). Al igual que en la clasificación de Zubizarreta, estos focos pueden reponerse a partir de un contexto interrogativo, por ejemplo:

(3) a. ¿Qué pasó? María compró un traje.

b. ¿Qué hizo María? María [F compró un traje].

c. ¿Qué compró María? María compró [F un traje].

Uno de los recursos más considerados en el estudio del foco es la prosodia. A pesar de que la prosodia sea el recurso de marcación de foco más estudiado y el que más relevancia tiene a la hora de identificar estructuras sintácticamente idénticas, existen en español otras herramientas lingüísticas para enfatizar segmentos de habla. La elección del orden de los constituyentes oracionales es una forma sintáctica de marcar el foco y así distinguir cuál es la parte más informativa del discurso y cuál la menos. Como indica Labastía (2006), el español fue caracterizado como una lengua de posición relativamente libre en cuanto a sus constituyentes y la estructura de la información está expresada principalmente a través del orden de palabras. El orden oracional no-marcado (4.a) no está regido por las funciones gramaticales de sujeto y objeto sino por los roles de los argumentos de los verbos que va de lo conocido a lo desconocido -el tema y el rema según la Escuela de Praga (Trubetzkoy, 1987) que corresponden a sujeto y predicado-. El orden marcado, en cambio, es el que en algún constituyente no tiene una posición canónica. Por ejemplo, la topicalización a la izquierda coloca al constituyente en la posición marcada, como se observa en (4.b)

(4) a. Juan dejó el libro ayer en la biblioteca.

b. En la biblioteca, Juan dejó el libro ayer.

Si bien el español parece ser una lengua de orden libre, en el sentido de que presenta mayor flexibilidad en las estructuras sintácticas (al igual que en el alemán y a diferencia del inglés), no es una lengua sin orden. Es decir, el orden no-marcado es claramente identificable incluso en estas lenguas. En las lenguas de orden rígido, como el inglés, es muy fácil identificar el orden no-marcado $\mathrm{y}$, en consecuencia, observar cuándo un quiebre de ese orden puede deberse a agramaticalidad y cuándo a una decisión pragmática del hablante (es decir, orden marcado). En las lenguas de orden libre, como el español y el alemán, identificar el orden no-marcado es más complejo. Una forma de determinarlo son las preguntas así de la nada, como “¿qué pasa?” (Gutiérrez Bravo, 2008). Son preguntas que corresponden a un contexto en el que el hablante que las emite no tiene conocimiento de la situación discursiva que está teniendo lugar. Típicamente la respuesta a este tipo de preguntas presenta el orden no-marcado de la lengua. Así como las preguntas así de la nada nos brindan la forma no marcada, se deduce que una interrogación especificada por un pronombre determinado (quién, dónde, cómo, etc.) informa un orden marcado en distintos constituyentes.

(5) a. ¿Quién dejó el libro en la biblioteca? [F Juan] dejó el libro en la biblioteca.

b. ¿Dónde dejó el libro Juan? Juan dejó el libro [F en la biblioteca]. 
En las interrogaciones, el pronombre ocuparía la posición sobre la cual se transmite la información nueva, por lo tanto estaría indicando no solo el foco en la respuesta sino también la parte de la pregunta que es informativamente relevante o que es necesario reponer.

En el estudio del foco en español, Toledo (1998) se propone caracterizar cuáles son los correlatos acústicos de las señales prosódicas. Para ello, utilizó un corpus de oraciones con estructura profunda similar, pero con distintas marcaciones focales según el contexto discursivo que surgía de una interrogación. Posteriormente, analiza contrastivamente la F0, la intensidad y duración de estas emisiones, con el fin de describir acústicamente la marcación focal. Los resultados muestran una variabilidad en el uso de estos patrones acústicos, lo que hace concluir que, en español, la marcación prosódica es asistemática y dependería más de una opción del hablante que del contexto.

Debido a la falta de consenso en la bibliografía acerca de los factores acústicos que inciden en la marcación focal, Face (2002) realiza un estudio experimental de la producción de foco en el español madrileño, con el fin de determinar el valor de la altura tonal para este fenómeno. Para ello, investiga la altura tonal de ciertas palabras, manipulando la posición oracional (inicial, media y final) en diferentes contextos focales. Los resultados muestran que, de acuerdo a la posición del foco, la altura tonal podría indicar varias cosas: dentro de una misma frase podría marcar que el hablante se está aproximando al foco de la misma o indicar la palabra focal por elevarse más que en una frase sin foco; después de la palabra focal, el hablante reduce el tono para marcar el final del foco. Si bien lo califica como complejo, el comportamiento de la altura tonal identifica que las variaciones en un mismo rasgo pueden comunicar un solo aspecto, el foco.

Por otra parte, Labastía (2006) realizó un análisis cualitativo del foco en el discurso espontáneo de un hablante del español de Buenos Aires, en el que tuvo en cuenta la F0, la duración y la intensidad. Los resultados concluyen que el español de Buenos Aires tiende a mantener el acento focal en el constituyente final, lo que genera que unidades enunciativas menores como las palabras sean resaltadas, en lugar de unidades más largas como las frases. Además, muestra que la ubicación del acento final puede tener una interpretación del foco contrastiva o no contrastiva.

Dorta (2008), por su parte, realiza un estudio multidimensional del foco en español en el que considera factores sintácticos, semánticos, pragmáticos, discursivos y prosódicos para describirlo. El análisis de un discurso oral espontáneo revela que si bien los recursos prosódicos son los más relevantes a la hora de señalar el foco, la marcación focal no siempre tiene como objetivo indicar información nueva, sino que depende de los intereses del hablante.

Si bien existen estudios en español acerca de la interacción entre la percepción de los datos acústicos y la expectativa lingüística (i.e. reconocimiento de la modalidad enunciativa, identificación del foco, prominencia silábica), estos no atendieron a los distintos aspectos involucrados de manera conjunta. En un estudio sobre el español madrileño, Face (2007) investigó experimentalmente el rol de las pistas acústicas en la percepción de las frases interrogativas y entonativas. Mediante la manipulación de la F0, se evaluó si un grupo de sujetos podía distinguir si un enunciado era interrogativo o declarativo a partir de oraciones completas y de otras incompletas. Los resultados muestran que los sujetos pueden reconocer acertadamente curvas entonacionales desde las primeras frases del enunciado y que pueden identificar de forma temprana cualquier alteración en la F0. Eso sugiere que esta pista acústica es la más relevante para el reconocimiento de la modalidad enunciativa. Por otra parte, en 
el estudio de la percepción del foco en el español, Lang-Rigal (2011) se propone identificar cuáles son los correlatos acústicos para la percepción del foco amplio y el foco estrecho en el español de Buenos Aires. Para ello, graba un solo enunciado ("Manolo viene mañana") con foco neutro (en toda la oración) y con foco estrecho (en el sujeto). Posteriormente, realizó una tarea de percepción en la que los sujetos debían indicar la palabra de mayor prominencia de la frase. Los resultados muestran que la percepción del foco estrecho en el sujeto ("Manolo") se debe a tres factores: un aumento tonal en la sílaba acentuada de esa palabra, el aumento de la duración de la misma sílaba y una pausa después de la palabra focalizada. Además, este estudio aporta evidencia de que la percepción del foco se ve afectada por las características acústicas de los elementos no focalizados.

En otras lenguas existen estudios que identifican los factores acústicos que intervienen en la percepción de la prominencia silábica. Eriksson et al. (2002) diseñaron una serie de experimentos en sueco con el objetivo de determinar hasta qué punto el esfuerzo vocal puede indicar la identificación subjetiva de la prominencia silábica. Los experimentos involucraban diferentes tests de percepción de enunciados en sueco con distintos grupos experimentales: un grupo de hablantes del sueco, otro de hablantes del inglés sin conocimiento alguno del sueco y, por último, un grupo de sujetos que debían evaluar la prominencia a partir de los enunciados escritos. Así, en un caso se evaluaba la percepción de los datos con interferencia lingüística, otro sin interferencia lingüística y la expectativa lingüística sin datos acústicos, respectivamente. Los resultados muestran que los hablantes del sueco y del inglés evalúan la prominencia de manera similar, aunque otorgando distinto peso a cada una de las pistas acústicas, mientras que las evaluaciones de las frases escritas (expectativa lingüística) fueron muy diferentes de las evaluaciones de las frases escuchadas. Los autores identifican así tres factores relevantes en la percepción acústica de la prominencia silábica: el esfuerzo vocal, el tono y la duración. Sin embargo, no logran establecer cuál de estos tres factores es el más relevante para la asignación de la prominencia. Las diferencias entre los resultados de las evaluaciones de los oyentes ingleses y de los suecos y las diferencias entre los que tuvieron acceso a los datos acústicos frente a los que no, indican que la información y las expectativas lingüísticas juegan un rol fundamental en la percepción de la prominencia y a veces esta percepción no tiene en cuenta los factores acústicos reales (procesamiento arriba-abajo o top-down).

Un estudio integral de todos estos fenómenos (reconocimiento de la modalidad, identificación del foco y percepción de la prominencia silábica) fue llevado a cabo por Mixdorff et al. (2015) para el alemán. En este trabajo, se exploró hasta qué punto la información lingüística como la modalidad, el foco y la prominencia silábica pueden ser extraídas de los rasgos prosódicos de un enunciado. Primeramente, se analizaron los rasgos prosódicos de diferentes enunciados que incluían dos modalidades (interrogativa y declarativa) y tres focos (neutro, sujeto y predicado). Se observaron la F0, la intensidad, la duración y calidad de voz y se los relacionó con las evaluaciones subjetivas de prominencia hechas por los sujetos. Se encontró que las partes focalizadas tienen rangos de F0 expandidos y un aumento de la intensidad y duración, y que las estructuras no focalizadas presentan el efecto contrario. Los resultados perceptuales parecen no coincidir enteramente con los rasgos acústicos. El foco amplio no fue correctamente identificado. Los sujetos elegían un foco por defecto (el último, lo que podría explicarse nuevamente con información lingüística).

En español, no contamos con estudios que se propongan analizar estos fenómenos (modalidad enunciativa, foco y prominencia silábica) sistemáticamente, de manera conjunta y tanto 
en percepción como en producción. Por ello, el presente trabajo se enmarca en un proyecto conjunto con el equipo alemán dirigido por Mixdorff (Department of Computer Science and Media, Beuth University Berlin) y propone seguir las mismas líneas de investigación que se iniciaron para el alemán, en español rioplatense. En este contexto, se considerará foco como el fragmento del enunciado producido por el hablante con mayor relevancia prosódica y prominencia como la percepción subjetiva de esta producción. Los objetivos del presente trabajo son:

a) determinar hasta qué punto los oyentes pueden reconocer la modalidad de un enunciado únicamente a partir de pistas prosódicas;

b) investigar la identificación correcta del foco por parte de los oyentes de acuerdo a pistas prosódicas;

c) observar la percepción subjetiva de la prominencia silábica en cada una de las condiciones focales;

d) correlacionar todas estas medidas subjetivas con los datos acústicos de las producciones orales (F0, duración, intensidad).

\section{Materiales y método}

Se utilizaron oraciones con la estructura: sujeto $(\mathrm{SN})$ y predicado $(\mathrm{V}+\mathrm{SP})$. El núcleo del SN y del SP presentaban variaciones en el acento léxico, que podía ser oxítono, paroxítono o proparoxítono. La combinación de cada una de estos sintagmas generó un total de nueve oraciones básicas (ver tabla 1). Las oraciones pertenecen al corpus AMPER (Atlas Multimedia de la Prosodia del Espacio Románico).

\begin{tabular}{|l|l|l|}
\hline El saxofón & \multirow{2}{*}{ se toca } & con obsesión \\
\cline { 1 - 1 } La guitarra & & con mesura \\
\cline { 1 - 1 } El triángulo & & con pánico \\
\hline
\end{tabular}

Tabla 1. Constituyentes de las oraciones y posibilidades de combinación.

Se les pidió a diez hablantes nativos de español, hablantes de la variedad rioplatense, (cinco mujeres y cinco hombres, sin problemas en su aparato fonatorio) que las reprodujeran con distintas variantes: con el foco en el sujeto, con el foco en el predicado y, por último, sin poner foco en ninguna parte de la oración. Se solicitó la realización de cada oración en dos modalidades: declarativa e interrogativa. El corpus final quedó compuesto por un total de 540 estímulos (cada hablante produjo nueve oraciones de base con tres focos en dos modalidades).

A partir de los estímulos obtenidos, fue diseñada una prueba experimental en la que los usuarios debían, en primer lugar, escuchar un audio, luego clasificar la modalidad oracional (declarativa, interrogativa o ambigua en caso que no tenga seguridad sobre la modalidad). Seguidamente, se les pedía que seleccionaran una de cinco preguntas que mejor se respondiera con esa frase (identificación del foco: foco neutro, en el sujeto, en el predicado o contrastivo) y, por último, se solicitó que para cada sílaba de la oración se señalara el nivel de prominencia en una escala del -5 al +5 . La marcación de la prominencia subjetiva generó 
así una curva para toda la oración (figura 1). En total evaluaron 547 oraciones, las primeras 7 funcionaron a modo de práctica. Participaron de este experimento 7 sujetos (cuatro hombres y tres mujeres) de alta escolaridad.

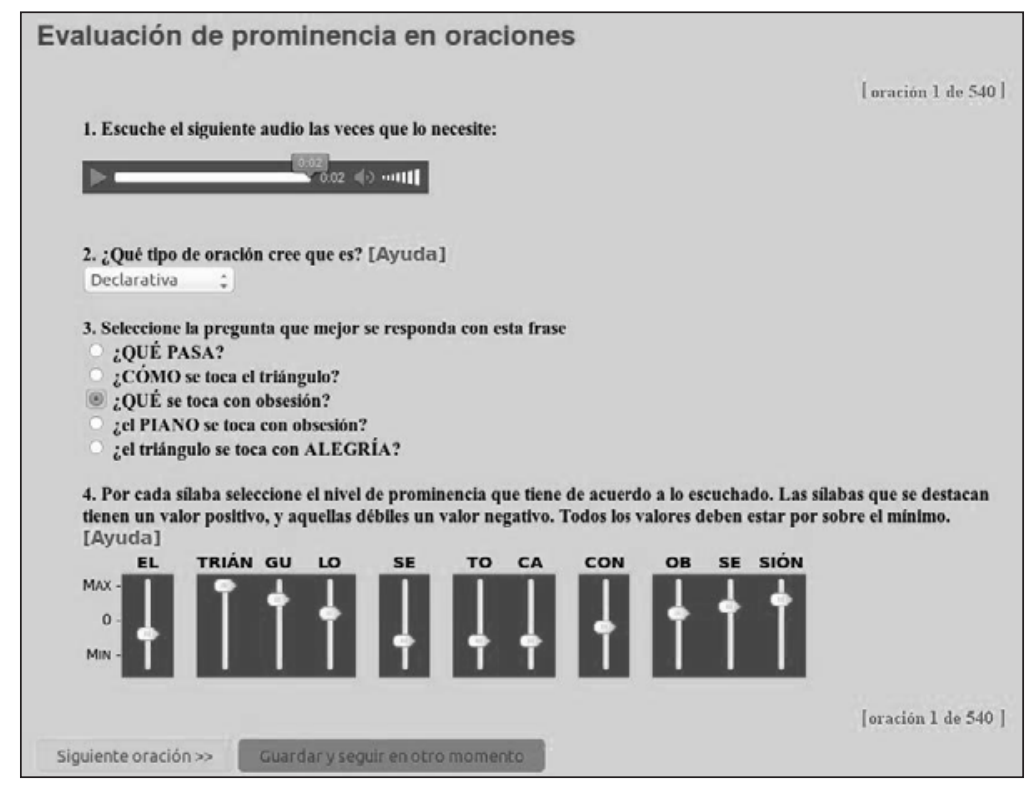

Figura 1. Estructura del experimento para la obtención de evaluaciones de prominencia de oraciones.

\section{Resultados}

En primer lugar, se extrajeron los porcentajes de aciertos en cuanto al tipo de oración (declarativa o interrogativa) (véase tabla 2). Como puede observarse, aunque en la modalidad declarativa las dificultades de determinación son menores, el porcentaje de aciertos en ambas modalidades es muy alto $(99,26 \%$ de aciertos en la modalidad declarativa y $97,13 \%$ en la modalidad interrogativa).

\begin{tabular}{|l|l|l|l|}
\cline { 2 - 4 } \multicolumn{1}{c|}{} & \multicolumn{2}{l|}{ Respuesta } \\
\hline Tipo de oración & Ambigua & Declarativa & Interrogativa \\
\hline Declarativa & 0 & 1894 & 14 \\
\hline Interrogativa & 14 & 41 & 1863 \\
\hline
\end{tabular}

Tabla 2. Respuesta por tipo de modalidad oracional.

En segundo lugar, se analizaron las respuestas para identificar el foco y los porcentajes de acierto en relación al tipo de foco y la modalidad de la oración. En la figura 2 se muestran los 
porcentajes de respuesta según la modalidad oracional. Puede observarse que las oraciones declarativas producidas sin foco son reconocidas mayoritariamente como oraciones con foco en el predicado $(61,69 \%)$ y al mismo tiempo las interrogativas sin foco fueron identificadas con foco en el sujeto o en predicado en igual proporción (43,01\% para cada tipo de respuesta). Tanto el foco en el sujeto como en el predicado fue reconocido correctamente en oraciones declarativas, sin embargo las oraciones interrogativas producidas con foco en el predicado presentan un porcentaje de confusión elevado, ya que el 31.36\% se identificó con foco en el sujeto.

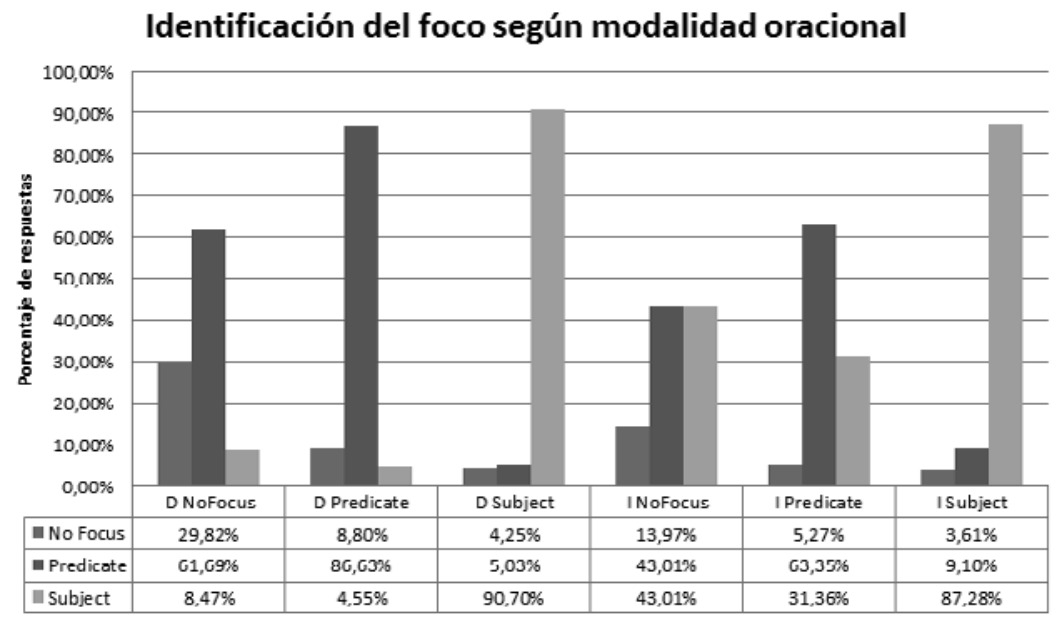

Figura 2. Porcentaje de tipo de respuesta en la identificación del foco en oraciones declarativas (D NoFocus, D Predicate y D Subject) y en oraciones interrogativas (I NoFocus, I Predicate e I Subject).

Por último, se observaron las prominencias silábicas señaladas por los sujetos (valores en el rango de $-5 \mathrm{a}+5$ ). Se tomó el valor de la prominencia máxima por sílaba dentro de la palabra nuclear del sujeto (segunda palabra de la oración) y del sintagma preposicional del predicado (sexta palabra de la oración). Los datos fueron normalizados con el fin de reducir un efecto de marcación inter sujeto. Se realizaron tests estadísticos para observar el valor de las prominencias percibidas en el núcleo del sujeto (palabra 2) y del sintagma preposicional del predicado (palabra 6), así como también la diferencia entre ambas, para cada una de las condiciones focales. Los resultados muestran que hay una diferencia significativa para la prominencia de la palabra 2 cuando el foco es producido en el sujeto, en relación a las otras condiciones (foco neutro, foco en el predicado) (Test de Wilcoxon para pares, $\mathrm{p}<0.01$ ). Al mismo tiempo, la prominencia de la palabra 6 (núcleo del sintagma preposicional del predicado) es significativamente mayor cuando el foco es producido en el predicado, frente al resto de las condiciones ( $\sin$ foco, foco en el sujeto) (Test de Wilcoxon para pares, $\mathrm{p}<<0.01$ ). Esto significa que las diferencias en las medias de prominencia percibida permiten separar a los distintos tipos de foco (foco en el sujeto, en el predicado o neutro). El foco producido por los hablantes puede separarse a partir de la diferencia entre la prominencia de la palabra nuclear del sujeto (palabra 2) y la del sintagma preposicional 
del predicado (palabra 6), mientras que el foco percibido y señalado por los evaluadores puede distinguirse a partir de la variable prominencia para la palabra nuclear del sujeto (palabra 2). Dichas diferencias pueden observarse en la figura 3.
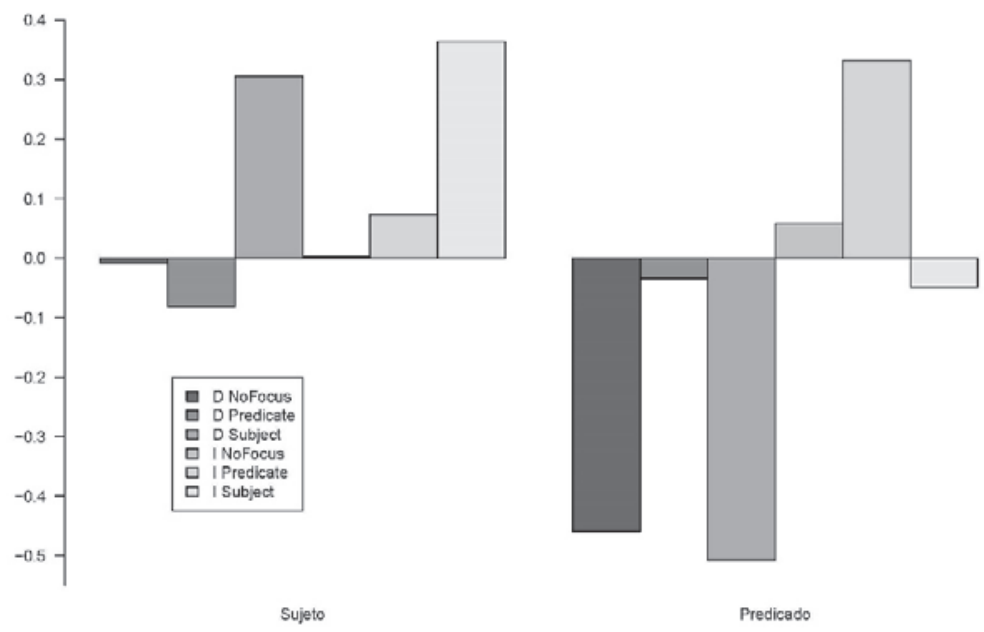

Figura 3. Z-score de la prominencia indicada en la palabra 2 (núcleo del sujeto) y en la palabra 6 (núcleo del sintagma preposicional del predicado) en todas las condiciones.

Por otra parte, se realizó un análisis de las prominencias en relación a la información acústica para el cual se extrajeron distintos parámetros acústicos para cada una de las sílabas. Posteriormente, se realizó un análisis estadístico para determinar el nivel de correlación entre cada uno de los parámetros acústicos con el nivel de prominencia asignado. El análisis de los datos acústicos revela una correlación entre prominencia y tres de los parámetros estudiados: duración, intensidad y F0 (Coeficiente de Correlación de Pearson 0.55, 0.31 y 0.32 respectivamente, $\mathrm{p}<<0.01)$.

\section{Discusión}

El presente trabajo se propuso determinar hasta qué punto los oyentes pueden reconocer la modalidad de una oración y la presencia de un segmento marcado o focalizado de manera específica únicamente a partir de pistas prosódicas.

En primer lugar, se observó que la modalidad oracional no presenta problemas de reconocimiento, ya que existe un alto porcentaje de aciertos y los casos ambiguos que se presentan ocurren solo en oraciones interrogativas. Este resultado es consistente con lo hallado en otros estudios de español madrileño (Face, 2007).

La identificación del foco presentó un comportamiento distinto de acuerdo a la modalidad enunciativa de las oraciones. Así, para las declarativas, la identificación del foco tuvo un porcentaje alto de aciertos cuando se encontraba en el sujeto o predicado $(90,70 \%$ y $86,63 \%$ respectivamente). Sin embargo, las declarativas de foco neutro presentaron problemas en su identificación, ya que el $61,69 \%$ de las oraciones con foco neutro fue señalada como una 
oración con foco en el predicado. La marcación del foco neutro como foco en el predicado para las declarativas puede deberse a que el foco neutro puede responder en igual medida a todas las preguntas, $y$, aunque con la misma entonación se reconocen distintos niveles de información y que lo informativo puede ir desde el todo (¿qué pasa?), al sujeto (¿qué se toca con obsesión?) o al predicado (¿cómo se toca el triángulo? en "El triángulo se toca con obsesión”), la información nueva se encuentra al final de la oración (que en este caso coincide con el predicado).

Por su parte, las oraciones interrogativas mostraron un alto porcentaje de acierto en la identificación del foco en el sujeto $(87,28 \%)$, mientras que se observaron confusiones para el reconocimiento del foco en el predicado y del foco neutro. El foco neutro de las interrogativas fue indicado tanto como foco en el sujeto o como foco en el predicado, con un mínimo porcentaje de aciertos $(13,97 \%)$. Esto parece indicar que dentro de una pregunta es difícil establecer un foco neutro o amplio en el sentido de que toda la pregunta se genere para indagar un segmento completamente desconocido, ya que lingüísticamente una pregunta se realiza sobre la base de algún conocimiento previo. Por otra parte, el foco producido en el predicado para las interrogativas presentó un alto porcentaje de errores, ya que los oyentes lo marcaron en un amplio número de casos como foco en el sujeto $(31,36 \%)$. Esto se podría deber a que en las interrogativas el foco está prototípicamente en la parte inicial (generalmente representado en el énfasis del pronombre) y la estructura de la información cambia según la modalidad. La forma de generar foco en una interrogación siempre involucra un desplazamiento a la izquierda del tipo “En la biblioteca estudió Juan?”, lo cual hace poco natural que el foco esté en la última parte de la interrogación. El oyente espera que el primer segmento oracional sea el que está focalizado o al menos el que esté actuando como interrogación (como ocurre en el caso de los pronombres o de los desplazamientos a la izquierda). Además, la entonación por defecto de las interrogaciones con F0 en aumento en el final (Navarro Tomás, 1967) interfiere en el procesamiento y podría obstaculizar la correcta identificación del foco.

Los resultados de foco en general parecen indicar que los evaluadores se basaron más en los aspectos informativos de la oración (información conocida seguida de información nueva) que en las pistas acústicas. Por ello, reconocieron foco neutro como foco en el predicado en las declarativas, en las que la información nueva se presenta de manera no marcada al final del enunciado, y de manera inversa para las interrogativas, la información solicitada se encuentra al comienzo del enunciado o sea en el lugar del pronombre. De manera general, se puede concluir que la modalidad del enunciado por las características default de la lengua interfiere en la identificación del foco. La información lingüística tiene un peso mayor que la información acústica lo que sugeriría un procesamiento de tipo arriba-abajo o top-down para la identificación del foco en español. Estos resultados coinciden con lo hallado por Eriksson para el sueco (2002).

El análisis de las prominencias silábicas marcadas por los evaluadores revela que, cuando el foco está en el sujeto, la prominencia máxima es marcada en la palabra nuclear del sujeto, y cuando el foco está en el predicado también se marca como más prominente el núcleo del sintagma focalizado en el predicado. Es decir, que la prominencia percibida permite separar los tipos de foco de acuerdo a cómo fueron producidos. Esto significa que, a diferencia de lo que ocurre con la identificación del foco en la percepción de la prominencia silábica, la información del estímulo acústico es más relevante que la información 
lingüística. Dicha relevancia puede observarse a partir del estudio de correlación entre la prominencia y las variables acústicas que arrojó una correlación directa y significativa entre la prominencia y la duración, la intensidad y F0.

Los resultados aquí expuestos nos permiten concluir que la identificación del foco a partir de preguntas está más sesgada por la información lingüística o por las estructuras informativas de la lengua (procesamiento arriba-abajo o top-down), mientras que la marcación de la prominencia silábica parece responder a la percepción directa del estímulo auditivo (procesamiento abajo-arriba o bottom-up).

\section{Referencias bibliográficas}

Dik, S. (1997). The Theory of Functional Grammar. Part 1: The Structure of the Clause. Berlin y New York: Mouton de Gruyter.

Dorta, J. (2008). "La focalización prosódica: funcionalidad en los niveles lingüístico y pragmático", Estudios de Fonética Experimental, XVII, págs. 105-138.

Eriksson, A., Grabe, E., \& Traunmüller, H. (2002). "Perception of syllable prominence by listeners with and without competence in the tested language", Speech Prosody, International Conference.

Face, T.L. (2002). "El foco y la altura tonal en español”, Boletín de Lingüística, 17, págs. 30-52.

Face, T.L. (2007). "The role of intonational cues in the perception of declaratives and absolute interrogatives in Castilian Spanish”, Estudios de fonética experimental, 16, págs. 186-225.

Gutiérrez Bravo, R. (2008). "La identificación de los tópicos y los focos", Nueva Revista de Filología Hispánica, LVI (2), págs. 363-401.

Halliday, M. A. K. (1967). Intonation and grammar in British English. The Hague: Mouton.

Labastía, L. O. (2006). "Prosodic prominence in Argentinian Spanish", Journal of Pragmatics: an Interdisciplinary Journal of Language Studies, 38 (10), págs. 1677-1705.

Ladd, D. (1983). "Phonological features of intonational peaks", Language, 59, págs. 721-759.

Lang-Rigal, J. (2011). "Perception of Narrow Focus Prosody in Buenos Aires Spanish”,Selected Proceedings of the 5th Conference on Laboratory Approaches to Romance Phonology, págs. 118-126.

Mixdorff, H., Cossio-Mercado, C., Hönemann A., Gurlekian J.A., Evin D. \& Torres, H. (2015). "Acoustic Correlates of Perceived Syllable Prominence in German". En: Proceedings of Interspeech (Ed).

Navarro Tomás, T. (1967). Manual de pronunciación española, sixth edition. New York: Hafner Publishing.

Toledo, G. (1998). "Señales prosódicas del foco", Revista Argentina de Lingüística, 5 (1-2), págs. 205-230.

Trubetzkoy, N. (1987). Principios de fonología. Madrid: Cincel.

Zubizarreta, M. L. (1999). "Las funciones informativas: tema y foco". En I. Bosque y V. Demonte (eds), Gramática descriptiva de la lengua española, Tomo 3 Entre la oración y el discurso, págs. 4215-4244. Madrid: Real Academia Española. 
